\title{
A Novel IncRNA ENST00000530525 Affects AN01 Contributing to Blood-Brain Barrier Injury in Cultured hCMEC/D3 Cells Under OGD/R Conditions.
}

\section{Wen Jiang}

Guangzhou University of Traditional Chinese Medicine: Guangzhou University of Chinese Medicine Jie Li

Guangdong Provincial Hospital of Traditional Chinese Medicine

\section{Yuefang Cai}

Guangzhou University of Traditional Chinese Medicine: Guangzhou University of Chinese Medicine

Wenchen Liu

Guangdong Provincial Hospital of Traditional Chinese Medicine

\section{Mei Chen}

Guangdong Provincial Hospital of Traditional Chinese Medicine

\section{Xiaoying Xu}

Sun Yat-Sen University

\section{Minzhen Deng}

Guangdong Provincial Hospital of Traditional Chinese Medicine

\section{Jingbo Sun}

Guangdong Provincial Hospital of Traditional Chinese Medicine

\section{Lihua Zhou}

Sun Yat-Sen University

\section{Yan Huang}

Guangdong Provincial Hospital of Traditional Chinese Medicine

\section{Shuang Wu}

Guangzhou University of Traditional Chinese Medicine: Guangzhou University of Chinese Medicine Xiao Cheng ( $\nabla$ chengxiaolucky@126.com )

Guangzhou University of Traditional Chinese Medicine: Guangzhou University of Chinese Medicine

\section{Research}

Keywords: Ischemic stroke, IncRNA, ENST00000530525, ANO1, oxygen-glucose deprivation and reperfusion, human cerebrovascular endothelial cells 
DOl: https://doi.org/10.21203/rs.3.rs-968571/v1

License: (c) (1) This work is licensed under a Creative Commons Attribution 4.0 International License. Read Full License 


\section{A novel IncRNA ENST00000530525 affects ANO1 contributing to blood-brain barrier injury in cultured hCMEC/D3 cells under OGD/R conditions}

Wen Jiang ${ }^{1 \#}$, Jie $\mathrm{Li}^{5}$, Yuefang $\mathrm{Cai}^{1}{ }^{1}$ Wenchen $\mathrm{Liu}^{2}$, Mei Chen ${ }^{2}$, Xiaoying $\mathrm{Xu}^{6}$, Minzhen Deng ${ }^{2}$, Jingbo Sun ${ }^{1,2,3,4}$, Li-hua Zhou ${ }^{6}$, Yan Huang ${ }^{1,2,3,4}$, Shuang Wu ${ }^{1}$, Xiao Cheng ${ }^{1,2,3,4 *}$

\section{Author details}

${ }^{1}$ Department of Second Institute of Clinical Medicine, Guangzhou University of Traditional Chinese Medicine, Guangzhou 510405, China; 12 Jichang Road, Guangzhou. ${ }^{2}$ Department of Neurology, Guangdong Provincial Hospital of Traditional Chinese Medicine/The Second Affiliated Hospital of Guangzhou University of Chinese Medicine, Guangzhou, 510120, China; 111 Dade Road, Guangzhou. ${ }^{3}$ State Key Laboratory of Dampness Syndrome of Chinese Medicine, The Second Affiliated Hospital of Guangzhou University of Chinese Medicine, Guangzhou 510120, China. ${ }^{4}$ Guangdong Provincial Key Laboratory of Research on Emergency in TCM, Guangzhou 510120, China. ${ }^{5}$ Department of Anesthesiology, Guangdong Provincial Hospital of Traditional Chinese Medicine/The Second Affiliated Hospital of Guangzhou University of Chinese Medicine, Guangzhou, 510120, China; 111 Dade Road, Guangzhou. ${ }^{6}$ Department of Anatomy, Sun Yat-Sen School of Medicine, Sun Yat-Sen University, Shenzhen, China.

\section{*Corresponding authors:}

Xiao Cheng, M.D., Ph.D.

Department of Neurology, Guangdong Provincial Hospital of Traditional Chinese Medicine; 111 Dade Road, Guangzhou, 510120, P.R. China.

Fax number: (86) 020-81887233

Telephone number: (86) 020-81887233-35948 
Email: chengxiaolucky@126.com

\begin{abstract}
Ischemic stroke (IS) is a major neurological disease with high fatality and residual disability burdens. Increasing amount of long noncoding RNAs (lncRNAs) have been revealed to play an important role in ischemic stroke. However, the roles and significances of most lncRNAs in ischemic stroke are still unknown. This study was performed to identify differentially expressed lncRNAs using a lncRNA microarray in whole blood samples of patients suffered from acute cerebral ischemia. Bioinformatics analyses including GO, KEGG pathway enrichment analysis, and proximity to putative stroke risk location analysis were performed.
\end{abstract} A novel lncRNA ENST00000530525 significantly decreased after ischemic stroke. Furthermore, we evaluated lncRNA ENST00000530525 expression in cultured hCMEC/D3 cells under oxygen-glucose deprivation/reoxygenation(OGD/R) conditions using fluorescent in situ hybridization (FISH) and quantitative real-time polymerase chain reaction (RT-qPCR) analysis. To investigate the function of lncRNA ENST00000530525, the plasmid of overexpression $(\mathrm{OE})$ and negative control(NC) were transfected into hCMEC/D3, then cell viability was detected by cell counting kit-8 (CCK-8) Assay after OGD/R,lncRNA ENST00000530525 and ANO1 expression were investigated using RT-qPCR and Immunofluorescence. For blood-brain barrier(BBB) permeability,FITC-dextran transendothelial permeability assay and Tight junction $(\mathrm{Tj})$ protein was detected.There were 3352 differentially expressed lncRNAs in blood samples of acute ischemic stroke patients. The validation results were consistent with gene chip data.GO and KEGG results showed these lncRNAs were mainly related to oxygen and glucose metabolism, leukocyte transendothelial migration,mitophagy and cellular senescence.Among these, lncRNA 
ENST00000530525 was the highly down-regulated lncRNA and mapped within the ischemic stroke associated gene anoctamin-1 (ANO1). We furtherly found lncRNA ENST00000530525 was down-regulated in hCMEC/D3 cells under 4h OGD and 20h reoxygenation(OGD4/R20) conditions. Up-regulating lncRNA ENST00000530525 decreased the cell viability while increased ANO1 expression and contributed to BBB injury of hCMEC/D3 cells after OGD4/R20.The lncRNA ENST00000530525 might plays deleterious roles in post-stroke pathogenesis. The results show light on some differentially expressed lncRNAs in human certainly participate through characteristic roles in post-stroke pathogenesis, thus, the roles and significances of some novel lncRNAs in ischemic stroke thereby warranting further study.

Keywords: Ischemic stroke, lncRNA, ENST00000530525, ANO1, oxygen-glucose deprivation and reperfusion; human cerebrovascular endothelial cells

\section{Introduction}

Stroke is a major neurological disease associated with high fatality and disability burdens. Ischemic stroke is most prevalent, accounting for up to $80 \%$ of all stroke cases [1]. Hypertension, diabetes, dyslipidaemia, atrial fibrillation, and obesity are quite prevalent among the Chinese aged 40 years and older[2]. This makes stroke a very important health issue.

Cerebral blood flow interruption kick-starts a cascade of complex and poorly understood pathophysiologic processes including oxidative stress, inflammation and apoptosis[3, 4]. Therefore, many scientists have turned to epigenetics to understand the molecular mechanisms, regulation of the complex stroke-induced pathophysiological processes as well as the elucidation of new therapeutic targets and the design of new treatment modalities $[5,6]$. The greatest theme so far is the alteration of the expression of some non-coding RNAs which 
could participate in post-stroke processes, both neuroprotective and neurodegenerative.

The long no-coding RNAs (lncRNAs) are more than 200 nucleotides long and do not encode any proteins. LncRNAs play important roles in ischemic stroke by modulating cell survival, inflammation, and angiogenesis[7]. They appear to perform these varied functions because they can interact with multiple elements on the central dogma of biology such as other RNAs, DNA, and proteins[8]. In recent years, a bulk of aberrantly expressed lncRNAs have been screened out in ischemic stroke patients or ischemia insulted animals using new technologies such as RNA-seq, deep sequencing, and microarrays[7]. However, the roles and significances of most lncRNAs in ischemic stroke remain poorly understood and warrant further study.

Therefore, this study was performed to evaluate lncRNA expression in whole blood samples of patients after acute cerebral ischemia. Bioinformatics analyses, including GO, KEGG pathway enrichment analysis and network analysis were also performed for the identified dysregulated genes to put forth predicted connections and functions of the identified differentially expressed lncRNAs. Furtherly we found lncRNA ENST00000530525 is highly decreased between stroke patients and controls, the high conservation of lncRNA ENST00000530525 implicates that its aberrant expression may play a critical role in stroke pathology. LncRNA ENST00000530525 is located intragenically with its neighbor gene ANO1. However, the role of lncRNA ENST00000530525 and regulatory mechanism among the lncRNA and ANO1 signal in stroke remains enigma. ANO1 (also known as transmembrane protein 16A,TMEM16A), is a molecular identify of calcium-activated chloride channels and widely expressed in eukaryotes. Recent studies have suggested that upregulated expression of ANO1 is associated with the occurrence and development of many diseases, including stroke [9-11]. Recently, it was found that ischemia stroke caused blood brain barrier(BBB) damage is accompanied by an increase in ANO1. Inhibition of ANO1 
significantly preserved BBB integrity by decreasing ICAM-A via the NF-kB signaling pathway[12]. ANO1 is strongly expressed in cerebrovascular endothelial cells, present study showed that ANO1 was contributing to the proliferation and migration of brain capillary endothelial cells, which is involved in $\mathrm{BBB}$ functions [13] .Based on the above, the underlying mechanisms and target of lncRNA ENST00000530525 in cultured hCMEC/D3 cells under OGD/R conditions were investigated, which may provide a better understanding of the functions and mechanisms of lncRNAs in ischemic stroke. Further elucidating the functions and mechanisms of these lncRNAs in biological systems under pathological conditions may lead to opportunities for identifying biomarkers and novel therapeutic targets of ischemic stroke.

\section{Methods}

\section{Study subjects}

10 patients with a diagnosis of ischemic stroke and were attended at the Guangdong Province Traditional Chinese Medical Hospital from September 2016 to November 2017 along with 20 control subjects were recruited to participate in this study. The study protocol was approved by ethics committee of Guangdong provincial hospital of Chinese medicine(B2016-149-01). Patients and their immediate caregivers or significant others provided written informed consent to participate after the details of the study were explained to them. There was no incentive to participate. Patient histories, examinations, and evaluations were collected after the acute ischemic stroke diagnoses were made by qualified neurologists. Standard stroke assessments performed at our hospital included Electrocardiograph(ECG) and Holter monitor, carotid Doppler, computed tomographic (CT) brain scan and magnetic resonance imaging, and magnetic resonance arteriogram of extracranial and intracranial vessels or computed tomographic angiography depending on clients' clinical data. Acute ischemic stroke patients who presented with rare pathogenesis other than atherosclerosis and cardiogenic stroke, 
psychopathology, pregnancy and lactating or those patients who are in other clinical trials when they suffered a stroke were all not eligible to participate in this study. The age and gender of the 20 control subjects were matched to the stroke patients and were without a history of stroke or did not have severe heart disease, cardiac insufficiency, hepatitis, renal insufficiency, respiratory failure, malignant tumor or gastrointestinal bleeding.

\section{Derivation and validation sets}

To validate differential lncRNA expression, 10 ischemic stroke patients and 20 control subjects of both sexes were chosen from all recruited patients and assigned randomly to either a derivation set or a validation set. All control samples used were matched for age, race, sex, and vascular risk factors, including hypertension, diabetes mellitus, and hyperlipidemia of the selected patients. Criteria for exclusion included previous stroke (for control subjects); pre-blood draw treatments with thrombolytics or anticoagulants; subarachnoid hemorrhage or hemorrhagic infarction; pre- or post-stroke infection; abuse of recreational drugs; dialysis; cancer; blood abnormalities or steroid and other immunosuppressive treatment. The derivation set was used to determine significant differentially expressed lncRNAs. The validation set for confirming the results of the microarray analysis by RT-qPCR. Primer sequences used are provided in Table S1.

\section{Blood collection and RNA isolation}

Venipuncture was performed once on all study participants and their whole blood drawn into EDTA tubes (Jingxin Biotechnology Co., Ltd, China). For stroke patients, it was performed after symptom onset, with times varying from a few hours to two weeks. For control samples, this time point was recorded as zero. Blood was snap frozen and stored at $-80^{\circ} \mathrm{C}$ until use. RNA isolation was performed by using Trizol (Invitrogen, USA), added into every 
cryopreserved serum supernatant sample according to the manufacturer's protocol.

\section{Array hybridization}

Arraystar Human lncRNA V4.0 chip (Arraystar, USA), which detects 40173 lncRNAs and 20730 protein-coding transcripts, was used. Sample marking and chip hybridization were based on the recommended experimental procedure of Agilent One-Color Microarray-Based Gene Expression Analysis (Agilent Technology, USA) with some modifications. Briefly, rRNA was removed from the total RNA to obtain mRNA using the mRNA-ONLYTM Eukaryotic mRNA Isolation Kit (Epicentre, USA). Each sample was amplified and transcribed into cRNA with fluorescence by random priming. The RNeasy Mini Kit (Qiagen 74104, German) was used to purify the marking cRNAs. Quantification of RNA was determined with a NanoDrop ND-1000 spectrophotometer (NanoDrop Technologies, ThermoFisher, USA), and quality assessment was made by an Agilent 2100 Bioanalyzer (Agilent, USA). Arrays were washed and processed on a GenePix 4000B chip scanner (Agilent, USA). Raw expression values (probe level data) for each gene were saved in.DAT file formats. The scanned results were transformed into digital data and then saved.

\section{Differential expression (DE) analysis}

To identify differentially expressed lncRNAs between ischemic stroke patients and the controls, differential expression analyses were performed using cuffdiff. Only the probes with a p-value of $<0.05$ and a fold-change higher than 2 were selected as the criteria for significantly differentially expressed lncRNA (DE lncRNA) or mRNAs (DE mRNA). To obtain an overview of the characteristics of lncRNA and mRNA expression profiles, heat maps were generated using hierarchical clustering analysis based on the normalized values of all DE lncRNAs using the R package. Bioinformatics analyses, including GO (the Gene 
Ontology project, http://geneontology.org/), KEGG (the database Kyoto Encyclopedia of Genes and Genomes) pathway enrichment analysis (http://www.kegg.jp/kegg/), and network analysis, were also performed for the identified dysregulated mRNAs. Further biological interpretation of the differentially expressed lncRNAs was examined manually by literature review and on the UCSC Genome Browser, NONCODE and OMIM ${ }^{\circledR}$-Online Mendelian Inheritance in Man ${ }^{\circledR}$ databases.

\section{Data normalization}

Agilent Feature Extraction (FE) Software (Agilent, USA) version 11.0.1.1 was used to get the original data. The original data was exported by GeneSpring GX v12.1 software (Agilent Technologies, USA). The data was normalized by preprocess Core in R package and proceed log2-transformation to get the final normalized data. The standardized data were further analyzed by screening high-quality probes which were marked Detected in at least three samples out of six. The lncRNAs expressed significantly different between two groups of samples were screened by Volcano Plots while the lncRNAs differentially expressed between two samples were screened by Fold Change. Lastly, hierarchical clustering was conducted using R script.

\section{hCMEC/D3 cells culture}

Human cerebral endothelial hCMEC/D3 cells were purchased from FuHeng Biology (FH1110, Shanghai, China), cultered in endothelial cell medium (ECM, ScienCell, Carlsbad, United States) with 10\% fetal bovine serum (FBS, Gibco, Australia) and incubate in a humidified atmosphere with $5 \% \mathrm{CO}_{2}$ at $37^{\circ} \mathrm{C}$.All experiments with hCMEC/D3 cells were used below 15 passages. 


\section{Oxygen-glucose deprivation/reoxygenation (OGD/R) treatment}

In order to mimic acute ischemic stroke in vitro, hCMEC/D3 cells were treated with OGD/R conditions. When the cells became confluent, culture mediums of the cells were replaced with glucose-free DMEM (Cienry, Zhejiang, China), and cells had been exposed to anaerobic conditions $\left(1 \% \mathrm{O}_{2}, 5 \% \mathrm{CO}_{2}, 94 \% \mathrm{~N} 2\right)$ for $2,4,6$ and 8 hours. After incubation, the cells were returned to regular medium and normal conditions for reoxygenation. The total time of oxygen glucose deprivation and reoxygenation is 24 hours.

\section{Cell counting kit-8 (CCK-8) assay}

Cell viability was evaluated using a cell counting kit-8 assay (Dojindo, Tokyo, Honshu, Japan). All the procedures followed the manufacturer's instructions.Cell suspensions were added $100 \mu 1$ to a 96 -well plate at a density of $1 \times 10^{4} / \mathrm{mL}$. After incubating with $\mathrm{NC}$ or OE $\left(\mathrm{MOI}=50_{\mathrm{n}} \mathrm{M}\right)$ in $96-$ well microtiter plates for $72 \mathrm{~h}$, a total of $10 \mu 1$ of CCK-8 solution was added to each well after OGD $4 \mathrm{~h}$ reoxygenation $20 \mathrm{~h}$. Finally, the absorbance at $450 \mathrm{~nm}$ was measured using a microplate reader (SYNERGY H1,BioTek), and five replicate wells were assessed in each experiment.

\section{RNA fluorescent in situ hybridization(FISH)}

Subcellular localization of the lncRNA ENST00000530525 was detected by the FISH Kit (RiboBio, Guangzhou, China) according to the manufacturer' s protocol. Cy3-labeled lncRNA ENST00000530525 probe and the control probe 18S were all designed by RiboBio (Guangzhou, China). Brifely, hCMEC/D3 were fixed in 4\% paraformaldehyde for $10 \mathrm{~min}$ at room temperature and washed three times with PBS for 5 min everytime.Cells were incubated in proteinase $\mathrm{K}$ for $5 \mathrm{~min}$ at $4{ }^{\circ} \mathrm{C}$ and washed three times with PBS for $5 \mathrm{~min}$ everytime.Then cells were incubated with a pre-hybridization buffer for $30 \mathrm{~min}$ at $37{ }^{\circ} \mathrm{C}$ and 
then incubated in hybridization buffer with specific probes for lncRNA ENST00000530525 and $18 \mathrm{~S}$ for $37{ }^{\circ} \mathrm{C}$ overnight.After hard and fast wash with SSC,DAPI was used for nucleus staining.Images were photographed using a fluorescence microscope(Nikon Ti2-E, Japan).

\section{Plasmid transfection}

The plasmid of overexpression lncRNA ENST00000530525 (OE) and negative control plasmid (NC) and transfection kit (riboFECT $\left.{ }^{\mathrm{TM}} \mathrm{CP}, \mathrm{C} 10511-05\right)$ were obtained from RiboBio company (Guangzhou, China). hCMEC/D3 cells were cultured and incubated for 3 days with OE or $\mathrm{NC}\left(\mathrm{MOI}=50_{\mathrm{n}} \mathrm{M}\right)$, which were then replaced by glucose-free DMEM for OGD 4 hours. The transfection efficiency of lncRNA ENST00000530525 was detected by RT-qPCR.

\section{FITC-dextran transendothelial permeability assay}

In brief, hCMEC/D3 cells were seeded onto polycarbonate 12-well transwell inserts with a $0.4 \mu \mathrm{m}$ pore size (Corning, USA) at a density of $4 \times 10^{4} \mathrm{cells} / \mathrm{cm}^{2}$. The cells were allowed to grow until they reached $70-80 \%$ confluence after plasmid transfection. Then $0.1 \mathrm{mg} / \mathrm{ml}$ FITC- labled dextran (MW, 70000, Sigma-Aldrich) was added to the upper chamber after OGD $4 \mathrm{~h}$ followed by $20 \mathrm{~h}$ reoxygenation, the lower chamber was filled with $500 \mu \mathrm{l}$ of PBS, and cells cultured for 5 minutes at $37^{\circ} \mathrm{C}$ under normoxic conditions. $100 \mu 1$ of supernatants from the lower compartment were analyzed by a microplate reader (excitation $490 \mathrm{~nm}$,emission $520 \mathrm{~nm}$ ).

\section{Quantitative real-time PCR for IncRNA and mRNA}

For RT-qPCR analysis, total RNA was isolated from the cells using TRIzol Reagent(Thermo Fisher Scientific,Waltham,Massachusetts,USA) following the manufacturer's protocol. Firststrand cDNA synthesis from $1 \mu \mathrm{g}$ of total RNA was performed using a reverse transcriptase 
cDNA synthesis kit (Takara, Dalian, China).RT-qPCR was conducted using SYBR ${ }^{\circledR}$ Premix Ex Taq ${ }^{\text {TM }}$ II (Takara, Dalian, China).The experiments were repeated at least three times independently to ensure the reproducibility of the results. Human $\beta$-actin gene was amplified as an internal control.Comparative quantification was done by using the 2- $\Delta \Delta \mathrm{Ct}$ method. Primer sequences used are provided in Table S1.

\section{Immunofluorescence}

For immunofluorescent staining, the cells were fixed with $4 \%$ paraformaldehyde at $4{ }^{\circ} \mathrm{C}$ overnight.After blocking with 5\%BSA,the cells were incubated with the following primary antibodies:rabbit monoclonal anti-ANO1 (1:200), rabbit monoclonal anti-ZO1 (1:200), rabbit anti-Occludin (1:100), rabbit anti-Claudin-5 (1:200), overnight at $4{ }^{\circ} \mathrm{C}$ overnight. The cells were washed with phosphate buffered saline(PBS) for 10 minutes and three times and then incubated with the indicated secondary antibodies for $1 \mathrm{~h}$ in the dark box at $37^{\circ}$ C.The antibodies and secondary antibodies Alexa Fluor 488 antibodies were all from Abcam.Thereafter, the cell nuclei were stained with DAPI (1:500,Beyotime).Images were photographed using a fluorescence microscope(Nikon Ti2-E, Japan).

\section{Statistical analysis}

All quantitative experiments were performed at least three times,and data was presented as mean \pm SD.Statistical tests were performed using the SPSS 24.0 statistical software and Graphed Prism 6. The comparisons about patient characteristics between the stroke group and the control group were determined by unpaired two-tailed Students's t-test (age) and chi-square test (gender, hypertension history, diabetes history, smoking history, cardiovascular history, and hyperlipidemia). The comparisons among multiple groups were analysed by one-way ANOVA. $P<0.05$ was considered statistically significant. 


\section{Results}

\section{Characteristics}

The characteristics of 10 stroke patients and 20 non-stroke cases are shown in table 1 . There are significantly more stroke patients with a positive history of hypertension, diabetes, and smoking.

Table 1 Demographics and distribution of comorbid conditions in the study sample.

\begin{tabular}{lll}
\hline Characteristics & $\begin{array}{l}\text { Non-Stroke Group } \\
(\mathrm{n}=20)\end{array}$ & $\begin{array}{l}\text { Stroke Group } \\
(\mathrm{n}=10)\end{array}$ \\
\hline Age (years) & $64.54 \pm 11.83$ & $63.58 \pm 10.20$ \\
Gender (Male) & $14(70 \%)$ & $8(80 \%)$ \\
Hypertension history & $10(50 \%)$ & $7(70 \%) *$ \\
Diabetes history & $1(5 \%)$ & $3(30 \%) *$ \\
Smoking history & $3(15 \%)$ & $4(40 \%) *$ \\
Cardiovascular disease history & $3(15 \%)$ & $1(5 \%)$ \\
Hyperlipidemia & $5(25 \%)$ & $5(5 \%)$
\end{tabular}

Table 1 The table shows the age of study participants along with the distribution of their comorbid conditions such as hypertension, diabetes, smoking, cardiovascular disease and hyperlipidemia. ${ }^{*} p<0.05$.

\section{Differentially IncRNAs expression and GO and KEGG analysis among ischemic stroke patients versus non-stroke patients}

We analyzed the differentially expressed lncRNA between ischemic stroke and non-stroke patients using arraystar Human lncRNA V4.0 chip. Unsupervised hierarchical clustering 
analysis generated from the expression profile of lncRNAs showed the different expression levels between stroke and control groups (Fig.1a). Volcano plots were used to show the statistically significant differences in lncRNAs expression between the two groups.3352 lncRNA transcripts were differently expressed between stroke and non-stroke groups, with 1318 up-regulated and 2034 down-regulated lncRNAs(Fig.1b). The top ten most significantly up and down-regulated lncRNAs are listed in Table 2. T120068 (fold change 12.2021) and ENST00000530525 (fold change -14.63) were the highly up-regulated and down-regulated lncRNAs, respectively.

The functions of differentially expressed lncRNAs was predicted using GO and KEGG pathway annotations of co-expressed mRNAs. The differentially expressed mRNAs in acute ischemic stroke were mainly related to ER membrane protein complax, Toll-like receptor binding,protein localization to nucleoplasm,RAGE receptor binding,intramolecular oxidoreductase activity,cellular glucuronidation,cellular glucuronation as well as $\mathrm{T}$ cell receptor complex(Fig.1c\&d).

The results of KEGG enrichment showed that the differential expressed lncRNAs were related to the pathway in pathophysiological processes underpinning acute cerebral infarction, such as pentose and glucuronate interconversions,regulation of actin cytoskeleton,RAS signal pathway, focal adhesion, leukocyte transendothelial migration,mitophagy as well as cellular senescence (Fig.1e\&f).

Table 2 The top 10 highly up- and down-regulated lncRNAs

a:

\begin{tabular}{lcccc}
\hline Seq-name & P-value & Fold Change & Regulation & Gene Symbol \\
\hline T120068 & 0.0010 & 12.2021 & up & G028344 \\
T346481 & 0.0045 & 11.6843 & up & G081575 \\
uc002vbb.3 & 0.0001 & 10.2441 & up & BC047484 \\
T333126 & 0.0001 & 10.2030 & up & G078089
\end{tabular}




\begin{tabular}{|c|c|c|c|c|}
\hline ENST00000530249 & 0.0001 & 9.8429 & up & CTD-3138F19.1 \\
\hline ENST00000597530 & 0.0007 & 9.3130 & up & RP11-251M1.1 \\
\hline $\mathrm{T} 224664$ & 0.0026 & 9.2702 & up & G051903 \\
\hline ENST00000447488 & 0.0019 & 9.0912 & up & AC073254.1 \\
\hline NR_046612 & 0.0071 & 8.7159 & up & HTR2A-AS1 \\
\hline T344288 & 0.0017 & 8.6688 & up & G080966 \\
\hline \multicolumn{5}{|l|}{$b:$} \\
\hline Seq-name & P-value & Fold Change & Regulation & Gene Symbol \\
\hline$\overline{\text { ENST00000530525 }}$ & 0.0001 & 14.6300 & down & RP11-805J14.3 \\
\hline T198099 & 0.0001 & 14.4622 & down & G045690 \\
\hline ENST00000523658 & 0.0016 & 14.0950 & down & RP11-402L5.1 \\
\hline T235708 & 0.0001 & 12.1118 & down & G054213 \\
\hline T328914 & 0.0002 & 11.0822 & down & G077138 \\
\hline NR_132119 & 0.0002 & 10.5270 & down & GTF2IP20 \\
\hline T024196 & 0.0001 & 9.8059 & down & G005224 \\
\hline ENST00000458252 & 0.0012 & 9.4884 & down & AC123886.2 \\
\hline T056136 & 0.0001 & 9.1733 & down & G012959 \\
\hline TCONS_00010056 & 0.0001 & 8.9389 & down & XLOC_004504 \\
\hline
\end{tabular}

Table 2 (a)The top ten most differentially up-regulated lncRNAs.(b)The top ten most differentially down-regulated lncRNAs

\section{Validation of differential IncRNAs expression}

To evaluate our gene chip performance, we measured the expression of 9 randomly selected but significantly dysregulated lncRNAs in the stroke group and non-stroke group using RT-qPCR analysis (Fig.2). There were seven up-regulated (ENST00000452599, ENST00000527450, ENST00000608826, T013651, T029143, T131416, T294865) and two down-regulated lncRNA transcripts (ENST00000530525, T058035). The result showed that the expression patterns of these lncRNAs were consistent with gene chip data in the stroke group and non-stroke group. 


\section{Biological interpretation-proximity to putative stroke genes}

Among the up-regulated lncRNAs, ASHGV40004037 is differentially expressed between stroke and control groups and is annotated to NR_046612 with genomic coordinates that maps to the putative ischemic risk loci, HTR2A, at cytoband 13q14.2. Similarly, ASHGV40013775 is annotated to NR_002312, another lncRNA that has genomic coordinates 45 kilo base pairs $(\mathrm{kb})$ downstream of the PARP2 cerebral ischemic injury-associated gene at 14q11.2 . ASHGV40056557 is expressed as NR_037932 which maps to the stroke implicated genes Apolipoprotein C and E within $420 \mathrm{~kb}$, while T111838 (ASHGV40056142) mapped at 14q32.33 within the metastasis-associated protein 1 (MTA1), a master chromatin modifier and transcriptional regulator associated genes. Also, T294865 (ASHGV40042838) mapped within $350 \mathrm{~kb}$ of another stroke associated gene ADAMTS2 at 5q35.3 while T297706 (ASHGV40043041) mapped to Sirtuin 5 (SIRT5) within 6kb at 6p23. Among the down-regulated lncRNAs, the annotation of ASHGV40008778 identified the validated lncRNA ENST00000530525 that is within the anoctamin-1 (ANO1), a chloride ion channel gene related to cerebrovascular remodeling. ENST00000395996 (ASHGV40001758) is within the VPS13B and associated with cerebral ischemic injury. Furthermore, NR_045414 (ASHGV40037155) at 4p16.3 mapped within 8kb of HTT and is associated with post-stroke depression (PSD) whilst ENST00000416061 (ASHGV40053990)'s genomic coordinates were $3.4 \mathrm{k}$ b downstream of SUV39H1, a histone methyltransferase related to stroke (Table3).

Table 3 Up- and down-regulated lncRNAs and proximity to putative stroke genes

\begin{tabular}{lllllll}
\hline Probe Name & IncRNA ID & Cyto-band & Gene & in IncRNA & IncRNA & FC \\
& & & Region & up-/down- & Distance(kb) \\
& & & & stream & from Gene \\
\hline ASHGV40004037 & NR_046612 & $13 q 14.2$ & HTR2A & within & within & 8.7159 \\
ASHGV40013775 & NR_002312 & 14q11.2 & PARP2 & down & 45 & 6.1135
\end{tabular}




$\begin{array}{lllllll}\text { ASHGV40056557 } & \text { NR_037932 } & \text { 19q13.32 } & \text { APOC2 } & \text { down } & 416.9 & 4.7449 \\ & & & \text { APOC4 } & \text { down } & 419.2 & \\ & & & \text { APOE } & \text { down } & 461.9 & \\ \text { ASHGV40056142 } & \text { T111838 } & \text { 14q32.33 } & \text { MTA1 } & \text { within } & \text { within } & 4.6273 \\ \text { ASHGV40042838 } & \text { T294865 } & 5 \mathrm{q} 35.3 & \text { ADAMTS2 } & \text { up } & 346.4 & 4.0617 \\ \text { ASHGV40043041 } & \text { T297706 } & 6 \mathrm{p} 23 & \text { SIRT5 } & \text { down } & 5.7 & 3.9144 \\ \text { ASHGV40008778 } & \text { ENST00000530525 } & 11 \mathrm{q} 13.3 & \text { ANO1 } & \text { within } & \text { within } & -14.6300 \\ \text { ASHGV40001758 } & \text { ENST00000395996 } & 8 \mathrm{q} 22.2 & \text { VPS13B } & \text { within } & \text { within } & -14.0950 \\ \text { ASHGV40037155 } & \text { NR_045414 } & 4 \mathrm{p} 16.3 & \text { HTT } & \text { down } & 7.6 & -6.3636 \\ \text { ASHGV40053990 } & \text { ENST00000416061 } & \text { XP11.23 } & \text { SUV39H1 } & \text { down } & 3.4 & -4.9941\end{array}$

Table 3 Significant $(p<0.05)$ differentially expressed lncRNA probe sets and those that changed over time in stroke vs control groups and proximity to putative stroke genes (Fold Change (FC) $\geq 2.0$, “-” means down regulated).

\section{LncRNA ENST00000530525 is located intragenically with ANO1}

LncRNA ENST00000530525 expression was significantly decreased after ischemia stroke, to futher research the function of lncRNA ENST00000530525 on stroke pathology,firstly the non-coding nature of lncRNA ENST00000530525 was confirmed by coding-potential analysis(Fig. S1)[14].Then we checked Ensembl[15], an online database to predict gene location[16],and found that the lncRNA ENST00000530525 is located intragenically with ANO1 (Fig.S2a). As we know, ANO1 is strongly expressed in cerebrovascular endothelial cells, present study showed that ANO1 was contributing to the proliferation and migration of brain capillary endothelial cells, which is involved in BBB functions[13]. We also checked STRING[17],an online database to summarize the network of predicted associations for a protein[18]. ANO1 was known to have strong links to epithelial ion channel such as cystic fibrosis transmembrane conductance regulator (CFTR) and Bestrophin family (Fig.S2b). Thus we furtherly speculate whether there is regulation of 1ncRNA ENST00000530525 and ANO1, which involved in the pathology of stroke. Because ANO1 is strongly expressed in 
cerebrovascular endothelial cells, so in the following study, hCMEC/D3 cells under OGD/R condition were chosed to mimic in vitro stroke model.

\section{LncRNA ENST00000530525 is down-regulated in hCMEC/D3 after OGD/R conditions}

To investigate lncRNA ENST00000530525 expression in hCMEC/D3 under OGD/R conditions, CCK-8 methods were used to test cell vitality. The results indicated that with the longer of OGD time, the worse of cell vitality (Fig.3a). Then we performed RT-qPCR to measure the expression of IncRNA ENST00000530525 in hCMEC/D3 after OGD/R injury. The results showed that the expression of lncRNA ENST00000530525 was significantly down-regulated in hCMEC/D3 compared with control after OGD/R conditions, which is the same as the results of chip sequencing in stroke patients (Fig.3b). And the expression of lncRNA ENST00000530525 was the highly down-regulated after 4h OGD and 20h reoxygenation.

Up-regulating IncRNA ENST00000530525 expression affects the cell viability of hCMEC/D3 under OGD/R injury

To explore the functions of lncRNA ENST00000530525 in hCMEC/D3 under OGD/R conditions, the lncRNA ENST00000530525 was up-regulated by plasmid transfection. The location of lncRNA ENST00000530525 was tested by FISH. The results showed that lncRNA ENST00000530525 mainly distributed in the cytoplasm as the control group-18S, which means plasmid transfection would effectively act on the lncRNA(Fig.4a).We furtherly detected the transfection efficiency by RT-qPCR. The result showed that the expression of lncRNA ENST00000530525 in OE group was effectively up-regulated by 28 folds as compared to that in the NC-treated cells after $72 \mathrm{~h}$ of transfection in hCMEC/D3, while there was no difference among the control and NC-treated groups (Fig.4b). It suggested that the 
transient plasmid transfection of lncRNA to cultured hCMEC/D3 could selectively up-regulate the lncRNA ENST00000530525 expression.

To further investigate whether over-expression lncRNA ENST00000530525 can influence the viability of hCMEC/D3 under OGD/R injury and the results showed that the cell viability was $1.00 \pm 0.05$ in untreated cells as a control, $0.65 \pm 0.03$ in normal control group, $0.62 \pm 0.03$ in NC-treated group, and $0.51 \pm 0.04$ in OE-treated group.Statistic analysis showed that the cell viability of OE-treated group was decreased compared to NC-treated group after OGD/R injury (Fig.4c), which indicated that over-expression of lncRNA ENST00000530525 to cultured hCMEC/D3 cells could led to more severe OGD/R ischemia injury.

\section{Up-regulating IncRNA ENST00000530525 increased the expression of ANO1}

To test whether up-regulation lncRNA ENST00000530525 will affect expressions of ANO1,RT-qPCR and immunofluorescence was used.The RT-qPCR results showed that the expression of ANO1 in OGD4/R20 group was up-regulated compared to control group.However the expression of ANO1 in OE group was effectively up-regulated by 4.27 folds as compared to that in the NC-treated cells after $72 \mathrm{~h}$ of transfection in hCMEC/D3, while there was no difference among the OGD4/R20 group and NC-treated group (Fig.5a).

Immunofluorescence results showed that protein expressions of ANO1 increased after OGD4/R20.Meanwhile when lncRNA ENST00000530525 was up-regulated by plasmid, ANO1 protein expression in OE group increased almost 2 folds compared to NC group, while there was no difference among the OGD4/R20 group and NC-treated group(Fig.5b).The reports demonstrated that altered expression of lncRNA ENST00000530525 could influence the expressions of ANO1 in hCMEC/D3 after OGD4/R20.

Targeted increasing of IncRNA ENST00000530525 deteriorated BBB Disruption after 


\section{OGD4/R20}

The effects of IncRNA ENST00000530525 increasement on BBB permeability were evaluated by FITC-dextran permeability.The results showed the FITC-dextran permeability in OGD4/R20 group was up-regulated compared to control group.However FITC-dextran permeability in OE group was effectively up-regulated by 1.41 folds as compared to that in the NC-treated cells after $72 \mathrm{~h}$ of plasmid transfection, while there was no difference among the OGD4/R20 group and NC group (Fig.6a).

Since BBB is mainly composed of endothelial cells that are connected by TJ proteins, we further evaluated the protein expression of ZO-1, occludin, and claudin-5 by immunofluorescence.After OGD4/R20, the expression levels of TJ proteins ( $\mathrm{ZO}-1$, occludin and claudin-5) were significantly down-regulated compared to control group. Meanwhile, IncRNA ENST00000530525 elevation decreased the protein levels of these three TJ proteins futher compared to NC group,while there was no difference among the OGD4/R20 group and NC group(Fig.6b-d). The results also showed that lncRNA ENST00000530525 elevation down-regulated the protein levels of ZO-1, occludin and claudin-5 after OGD4/R20.

\section{Discussion}

In current study,we revealed that lncRNA ENST00000530525 were down-regulated in OGD4/R20 induced ischemic stroke cell model, while ANO1 was up-regulated.ANO1 were previously reported to be up-regulated in ischemic stroke, and destroyed BBB integrity, via regulating the $\mathrm{NF}_{-\mathrm{k}} \mathrm{B}$ signaling pathway[12]. Our finding was consistent with these reports.It was noteworthy that when IncRNA ENST00000530525 was up-regulated by plasmid,the RNA and protein expressions of ANO1 was also increased,while the cell vitality of 
hCMEC/D3 and BBB integrity was inhibited.These evidences indicated that lncRNA ENST00000530525 might affect its neighbor gene ANO1 to play an important role in BBB integrity(Fig.7).

Stroke triumphs many non-communicable conditions in causing death and disability worldwide. However, the narrow therapeutic window of its standard therapy and difficulty making a quick diagnosis in most under-resourced medical facilities (no brain imaging), necessitates the need to understand the molecular regulation of stroke and identification of its biomarkers. Such knowledge would enable the identification of quick biomarkers among differentially expressed proteins and mRNAs as well as provide novel insights into the mechanisms of stroke pathogenesis in patients[19, 20]. Consequently, this study identified several stroke-induced changes in the expression of some circulating lncRNAs and their probable target of genes.

Studies in rodents revealed the mechanisms of action of some of the dysregulated lncRNAs. As we know there were four archetypes of molecular functions that lncRNAs execute-as signals, decoys, guides, and scaffolds[21]. For instance, cis-regulation ,which means lncRNA can directly bind to adjacent DNA in chromosomes and regulate its expression,is one kind of signals and plays a crucial role in stroke[22].lncRNA Peril act in this manner to positively regulate the expression of two genes in a 1.5 million base pairs $(\mathrm{Mb})$ away from its transcription location[23]. Accordingly, it is possible that other cis-lncRNAs may regulate cerebral ischemic stroke genes this way as several significantly differentially expressed lncRNAs in our study mapped within or to the neighboring genes associated with ischemic stroke were listed (Table3).

Then we hypothesized that lncRNA ENST00000530525 might affect its neighbor gene ANO1 though cis-regulation.To vertify our hypothesis, double luciferase assay was used.Briefly,we construct plasmid of Luc-ANO1-NC,Luc-ANO1-mimic, lncRNA-NC, 
lncRNA-OE.Then we transfected these plasmid into $293 \mathrm{~T}$ cells.After $72 \mathrm{~h}$,we detected firefly luminescence and renilla luminescence.However,There was no significant difference between Luc-ANO1-NC and Luc-ANO1-mimic when lncRNA ENST00000530525 was overregulated $(\mathrm{P}<0.05$ but the difference was less than $20 \%)$, indicating that lncRNA could not bind to target gene ANO1 and could not cis-act its expression(Fig.S3).Thus the lncRNA may regulate the target gene ANO1 though other mechanism,such as decoys.Recent studies have provided intriguing evidence for decoys lncRNA.LncRNA U90926 directly bound to malate dehydrogenase 2 (MDH2) and competitively inhibited the binding of MDH2 to the CXCL2 3' untranslated region (UTR), thus protecting against MDH2-mediated decay of CXCL2 mRNA[24].In our study,which mechanism lncRNA ENST00000530525 affectted its target gene ANO1 may need more research.

In the up-regulated lncRNAs, NR_002312 at 14q11.2 is 45kb away from Poly(ADP-ribose) polymerase-2 (PARP2). PARP2 is responsive to DNA damage and drives cell death pathways in ischemic stroke[25]. Also, lncRNA, NR_037932 (upregulated) was mapped to APOC2 and APOC4 within $420 \mathrm{~kb}$ and APOE within $462 \mathrm{~kb}$ at 19q13.32. APOE is central to the transport and metabolism of lipids and plays an important role in both cerebral ischemic stroke and coronary heart disease[26]. APOC4 correlates with stroke recovery whilst APOC2 is involved in the pathophysiology of post-stroke depression[27, 28]. These associations may be key to the functions of the lncRNAs in the evolution of stroke and can serve as pointers to biomarkers if pursued further. Another significant differentially expressed lncRNA, T297706, is within $6 \mathrm{~kb}$ of SIRT5 at $6 \mathrm{p} 23$ which increases blood-brain barrier permeability by degrading occludins [29] as well as promote arterial thrombosis via endothelial PAI-1 expression [30].

Among the down-regulated lncRNAs, ENST00000395996 is within vacuolar protein sorting 13 homolog B (VPS13B). VPS13B plays participates in the pathoevolution of 
atherosclerosis-induced ischemic stroke[31]. The HTT gene is less than $8 \mathrm{~kb}$ away from the down-regulated lncRNA NR_045414 while the up-regulated lncRNA is within the HTR2A gene. Both HTT and HTR2A are associated with post-stroke disease[32]. The highly down-regulated lncRNA ENST00000530525 is within the anoctamin-1(ANO1) gene. ANO1 (or TMEM16A), a chloride $\left(\mathrm{Cl}^{-}\right)$channel gene, is mainly overexpressed in mice cerebral endothelial cells after ischemic stroke. Inhibiting ANO1 attenuated brain infarct size and neurological deficits as well as preserved blood brain barrier integrity[12]. Therefore, it is plausible that the lncRNA ENST00000530525 may affect the expression of ANO1 gene by cis-regulation after ischemic stroke due to the proximity.

Due to their relatively recent discovery, the expression and functions of the majority of lncRNAs in the post-stroke brain are largely unknown. However, numerous lncRNAs are emerging as important regulators of transcription and translation. These findings of dysregulated lncRNAs may help to guide evidence-based preventive measures and the search for a cure. Further studies will be needed to establish whether these remaining lncRNAs can modulate stroke associated genes by cis-regulation in vivo or vitro.

\section{Declarations}

\section{Abbreviations}

IS:Ischemic stroke;IncRNAs:Long noncoding RNAs ;OGD/R:Oxygen-glucose deprivation/ reoxygenation;FISH:Fluorescent in situ hybridization; RT-qPCR: Quantitative real-time polymerase chain reaction; OE: Overexpression;NC:Negative control;CCK-8:cell counting kit-8;BBB:Blood-brain barrier;Tj:Tight junction;ANO1:Anoctamin-1;TMEM16A: Transmembrane protein 16A;ECG: Electrocardiograph;CT:Computed tomographic; 
DE:Differential expression;FE:Feature Extraction;CFTR:Cystic fibrosis transmembrane conductance regulator;ORF:Open reading frame;CSF:Codon substitution frequency

\section{Ethics approval and consent to participate}

All human experiments were approved by and conformed to ethics committee of Guangdong provincial hospital of Chinese medicine(B2016-149-01).

\section{Consent for publication}

Not applicable.

\section{Availability of data and materials}

The datasets used and/or analysed during the current study are available from the corresponding author on reasonable request.

\section{Competing interests}

The authors declare that they have no conflict of interest.

\section{Funding}

This study was financially supported by the National Natural Science Foundation of China, No. 81303115, 81774042, 81771353 and 81904104; the Pearl River S \& T Nova Program of Guangzhou, No.201806010025; the Youth Pilot Project of Chinese Society of Traditional Chinese Medicine, No. CACM-2018-QNRC2-C09; the Science and Technology Program of Guangzhou City of China, No. 201604020003 and202102010268;Guangdong Provincial Key Laboratory of Research on Emergency in TCM, No.2017B030314176; 2018-75 and 2019-140; the Administration of Traditional Chinese Medicine of Guangdong Province, 
No.20211203;Postdoctoral Science Foundation of China, No.2021M690759. The Specialty Program of Guangdong Province Hospital of Traditional Chinese Medicine of China, No. YN2016MJ07, 2015KT1294, YN2015MS02, and YN2018ZD07.

\section{Authors' contributions}

W. J. performed the experiments, data statistics, and was a major contributor in writing the manuscript.J.L. and Y.C. participated in drafting and revised the manuscript.W.L. analyzed and interpreted the patient data regarding the stroke patients.M.C.,X.X., M.D. and W.S. carried out experiments and analyzed the data. J.S.,L.Z.,Y.H. and X.C. supervised the design of the study and reviewed this manuscript. All authors reviewed and approved the final version of this paper.All authors read and approved the final manuscript.

\section{Acknowledgements}

Not applicable.

\section{References}

1. Wang W, Jiang B, Sun H, Ru X, Sun D, Wang L, et al. Prevalence, incidence, and mortality of stroke in china: Results from a nationwide population-based survey of 480687 adults. Circulation. 2017;135:759-771

2. Li Q, Wu H, Yue W, Dai $Q$, Liang $H$, Bian $H$, et al. Prevalence of stroke and vascular risk factors in china: A nationwide community-based study. Sci Rep. 2017;7:6402

3. Schaukowitch K, Kim TK. Emerging epigenetic mechanisms of long non-coding rnas. Neuroscience. 2014;264:25-38

4. Ren W, Yang X. Pathophysiology of long non-coding rnas in ischemic stroke. Frontiers in molecular neuroscience. 2018;11:96

5. Dharap A, Nakka VP, Vemuganti R. Effect of focal ischemia on long noncoding rnas. Stroke. 2012;43:2800-2802

6. Liu C, Yang J, Zhang C, Liu M, Geng X, Ji X, et al. Analysis of long non-coding rna expression profiles following focal cerebral ischemia in mice. Neuroscience letters. 2018;665:123-129

7. Bao MH, Szeto V, Yang BB, Zhu SZ, Sun HS, Feng ZP. Long non-coding rnas in ischemic stroke. Cell death \& disease. 2018;9:281

8. Akella A, Bhattarai S, Dharap A. Long noncoding rnas in the pathophysiology of ischemic stroke. Neuromolecular medicine. 2019;21:474-483 
9. Deng L, Yang J, Chen H, Ma B, Pan K, Su C, et al. Knockdown of tmem16a suppressed mapk and inhibited cell proliferation and migration in hepatocellular carcinoma. OncoTargets and therapy. 2016;9:325-333

10. Wang $M$, Yang $H$, Zheng $L Y$, Zhang $Z$, Tang $Y B$, Wang $G L$, et al. Downregulation of tmem16a calcium-activated chloride channel contributes to cerebrovascular remodeling during hypertension by promoting basilar smooth muscle cell proliferation. Circulation. 2012;125:697-707

11. Wu MM, Lou J, Song BL, Gong YF, Li YC, Yu CJ, et al. Hypoxia augments the calcium-activated chloride current carried by anoctamin-1 in cardiac vascular endothelial cells of neonatal mice. British journal of pharmacology. 2014;171:3680-3692

12. Liu PY, Zhang Z, Liu Y, Tang XL, Shu S, Bao XY, et al. Tmem16a inhibition preserves blood-brain barrier integrity after ischemic stroke. Frontiers in cellular neuroscience. 2019;13:360

13. Suzuki T, Yasumoto M, Suzuki Y, Asai K, Imaizumi Y, Yamamura H. Tmem16a ca(2+)-activated $\mathrm{cl}(-)$ channel regulates the proliferation and migration of brain capillary endothelial cells. Molecular pharmacology. 2020;98:61-71

14. https://asia.ensembl.org/index.html

15. Abdillahi M, Ananthakrishnan R, Vedantham S, Shang L, Zhu Z, Rosario R, et al. Aldose reductase modulates cardiac glycogen synthase kinase- $3 \beta$ phosphorylation during ischemia-reperfusion. American journal of physiology. Heart and circulatory physiology. 2012;303:H297-308

16. Yates AD, Achuthan P, Akanni W, Allen J, Allen J, Alvarez-Jarreta J, et al. Ensembl 2020. Nucleic acids research. 2020;48:D682-d688

17. https://string-db.org/

18. Szklarczyk D, Franceschini A, Wyder S, Forslund K, Heller D, Huerta-Cepas J, et al. String v10: Protein-protein interaction networks, integrated over the tree of life. Nucleic acids research. 2015;43:D447-452

19. Falcione S, Kamtchum-Tatuene J, Sykes G, Jickling GC. Rna expression studies in stroke: What can they tell us about stroke mechanism? Current opinion in neurology. 2020;33:24-29

20. An integrated encyclopedia of DNA elements in the human genome. Nature. 2012;489:57-74

21. Wang KC, Chang HY. Molecular mechanisms of long noncoding rnas. Molecular cell. 2011;43:904-914

22. Yan P, Luo S, Lu JY, Shen X. Cis- and trans-acting Incrnas in pluripotency and reprogramming. Current opinion in genetics \& development. 2017;46:170-178

23. Groff AF, Barutcu AR, Lewandowski JP, Rinn JL. Enhancers in the peril lincrna locus regulate distant but not local genes. Genome biology. 2018;19:219

24. Chen J, Jin J, Zhang X, Yu H, Zhu X, Yu L, et al. Microglial Inc-u90926 facilitates neutrophil infiltration in ischemic stroke via mdh2/cxcl2 axis. Molecular therapy : the journal of the American Society of Gene Therapy. 2021;29:2873-2885

25. Kofler J, Otsuka T, Zhang Z, Noppens R, Grafe MR, Koh DW, et al. Differential effect of parp-2 deletion on brain injury after focal and global cerebral ischemia. J Cereb Blood Flow Metab. 2006;26:135-141

26. Satizabal CL, Samieri C, Davis-Plourde KL, Voetsch B, Aparicio HJ, Pase MP, et al. Apoe and the association of fatty acids with the risk of stroke, coronary heart disease, and mortality. Stroke. 2018;49:2822-2829

27. Plubell DL, Fenton AM, Rosario S, Bergstrom P, Wilmarth PA, Clark WM, et al. High-density lipoprotein carries markers that track with recovery from stroke. Circ Res. 2020;127:1274-1287

28. Zhan Y, Yang YT, You HM, Cao D, Liu CY, Zhou CJ, et al. Plasma-based proteomics reveals lipid metabolic and immunoregulatory dysregulation in post-stroke depression. European psychiatry : the journal of the Association of European Psychiatrists. 2014;29:307-315 
29. Diaz-Cañestro $C$, Merlini M, Bonetti NR, Liberale L, Wüst $P$, Briand-Schumacher $S$, et al. Sirtuin 5 as a novel target to blunt blood-brain barrier damage induced by cerebral ischemia/reperfusion injury. Int J Cardiol. 2018;260:148-155

30. Liberale L, Akhmedov A, Vlachogiannis NI, Bonetti NR, Nageswaran V, Miranda MX, et al. Sirtuin 5 promotes arterial thrombosis by blunting the fibrinolytic system. Cardiovascular research. 2020

31. Ruan W, Wu J, Su J, Jiang Y, Pang T, Li J. Altered Incrnas transcriptomic profiles in atherosclerosis-induced ischemic stroke. Cellular and molecular neurobiology. 2020

32. Kim JM, Stewart R, Bae KY, Kim SW, Kang HJ, Shin IS, et al. Serotonergic and bdnf genes and risk of depression after stroke. Journal of affective disorders. 2012;136:833-840

\section{Figure legend}

Fig.1 LncRNAs expression and GO and KEGG analysis among ischemic stroke patients versus non-stroke controls (a) Hierarchical Clustering shows a distinguishable lncRNA expression profile expression profile. (b) Volcano plot of the differentially expressed lncRNAs in stroke patients and non-stroke controls. Red points in the plot represent statistically significant differentially up-expressed lncRNAs, while the green represent down-expressed lncRNAs. (c)GO terms for the differentially expressed up-regulated mRNAs.(d)GO terms for the differentially expressed down-regulated mRNAs. (e) KEGG terms for the differentially expressed up-regulated mRNAs. (f) KEGG terms for the differentially expressed down-regulated mRNAs.

Fig.2 Validation of differential lncRNA expression.The expression level of the selected lncRNAs in the stroke group and non-stroke group. qPCR was used to verify differentially expressed lncRNAs $(p<0.05)$. The blue points represent $q P C R$ and the red points represent the gene chip of RNA-seq. $n=6$.

Fig.3 LncRNA ENST00000530525 is down-regulated in hCMEC/D3 after OGD/R.Cells were exposed to OGD for $2,4,6$ or $8 \mathrm{~h}$ and returned to room air (reoxygenation) for $22,20,18$ or $16 \mathrm{~h}$. Each bar is labeled with the duration of OGD followed by the period of reoxygenation (e.g.,OGD2/R22 refers to $2 \mathrm{~h}$ of OGD and $22 \mathrm{~h}$ of reoxygenation).(a)The cell 
vitality was get more and more worse while the OGD time was rising.(b)The lncRNA highly down-regulated on the condition of OGD4/R20. For each treatment, the data are obtained

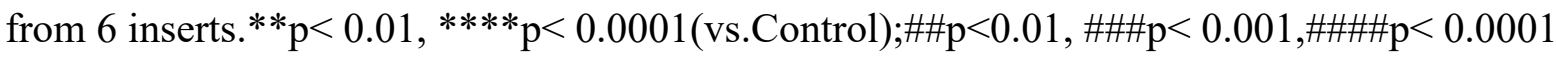
(vs. OGD4/R20),n=6,one-way ANOVA.

Fig.4 Up-regulating the Expression of lncRNA ENST00000530525 Affects the cell vitality of hCMEC/D3.(a)FISH showned that ENST00000530525 mainly distributed in the cytoplasm as the control group-18S.Scale bar: $50 \mu \mathrm{m}$.(b)The transduction efficiency detected by RT-qPCR.(c)CCK-8 assay revealed cell vitality.****p $<0.0001$ (vs.Control);\#\#\#p $<$ 0.001,\#\#\#p $<0.0001$ (vs. NC),n=6,one-way ANOVA.

Fig.5 Up-regulating lncRNA ENST00000530525 increased the expression of ANO1.The effects of lncRNA ENST00000530525 up-regulation by plasmid on gene and protein expressions of ANO1 in hCMEC/D3 after OGD4/R20 condition. (a)RT-qPCR results show that ANO1 increased after OGD4/R20, which increased more significantly when lncRNA ENST00000530525 was up-regulated by plasmid.(b)The immunofluorescence results show that transfecting a plasmid in hCMEC/D3 resulted in increasement of ANO1 protein expressions. ${ }^{* *} \mathrm{p}<0.01,{ }^{* * * *} \mathrm{p}<0.0001$ (vs.Control);\#\#\# $\mathrm{p}<0.0001$ (vs. NC), $\mathrm{n}=6$,one-way ANOVA.

Fig.6 Increasing lncRNA ENST00000530525 deteriorated OGD/R-induced transendothelial permeability.(a)Quantification of transendothelial permeability detected by dextran leakage.(b-d)Representative immunofluorescence results of ZO-1, occludin and claudin-5 after OGD/R treatments. ${ }^{* * * *} \mathrm{p}<0.0001$ (vs.Control);\#p $<0.001, \# \# \mathrm{p}<0.0001 ; \# \# \# \mathrm{p}<$ 0.001,\#\#\#\#<0.0001 (vs. NC),n=6,one-way ANOVA. 
Fig.7 Schematic diagram of this paper.This study was performed to identify differentially expressed lncRNAs using a lncRNA microarray in whole blood samples of patients suffered from acute cerebral ischemia.There were 3352 lncRNA transcripts differently expressed between the blood plasma of stroke and non-stroke patients, with 1318 up-regulated and 2034 down-regulated lncRNAs using arraystar Human lncRNA V4.0 chip.Meanwhile the lncRNA ENST00000530525 was the highly down-regulated lncRNA and ANO1 was its proximity to putative stroke gene.Up-regulating lncRNA ENST00000530525 would improve the expression of ANO1, which would destroy BBB integrity further,in hCMEC/D3 after OGD4/R20. 
Figures
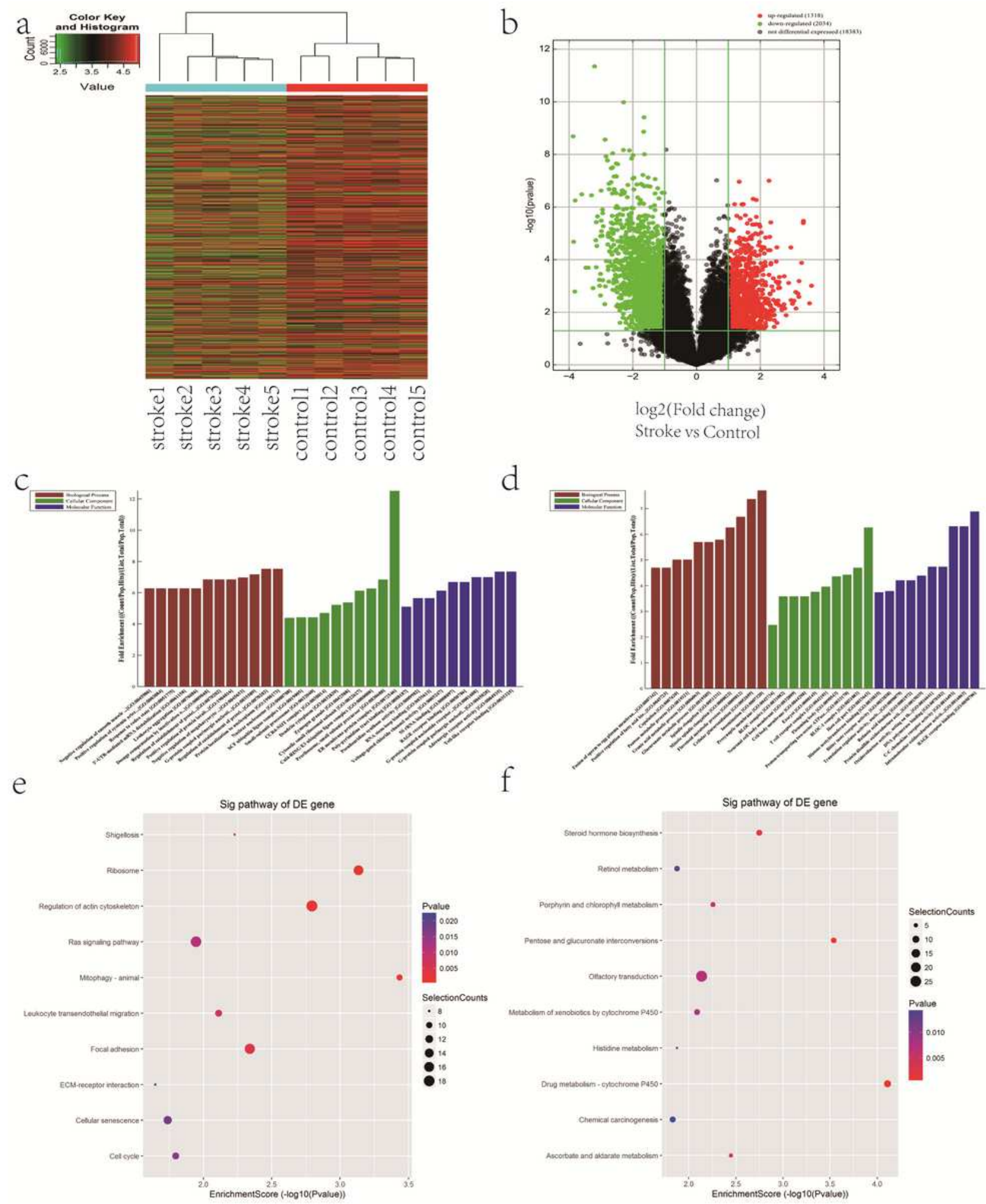

Figure 1

LncRNAs expression and GO and KEGG analysis among ischemic stroke patients versus non-stroke controls (a) Hierarchical Clustering shows a distinguishable IncRNA expression profile expression profile. (b) Volcano plot of the differentially expressed IncRNAs in stroke patients and non-stroke controls. Red 
points in the plot represent statistically significant differentially up-expressed IncRNAs, while the green represent down-expressed IncRNAs. (c)GO terms for the differentially expressed up-regulated mRNAs. (d)GO terms for the differentially expressed down-regulated mRNAs. (e) KEGG terms for the differentially expressed up-regulated mRNAs. (f) KEGG terms for the differentially expressed down-regulated mRNAs.

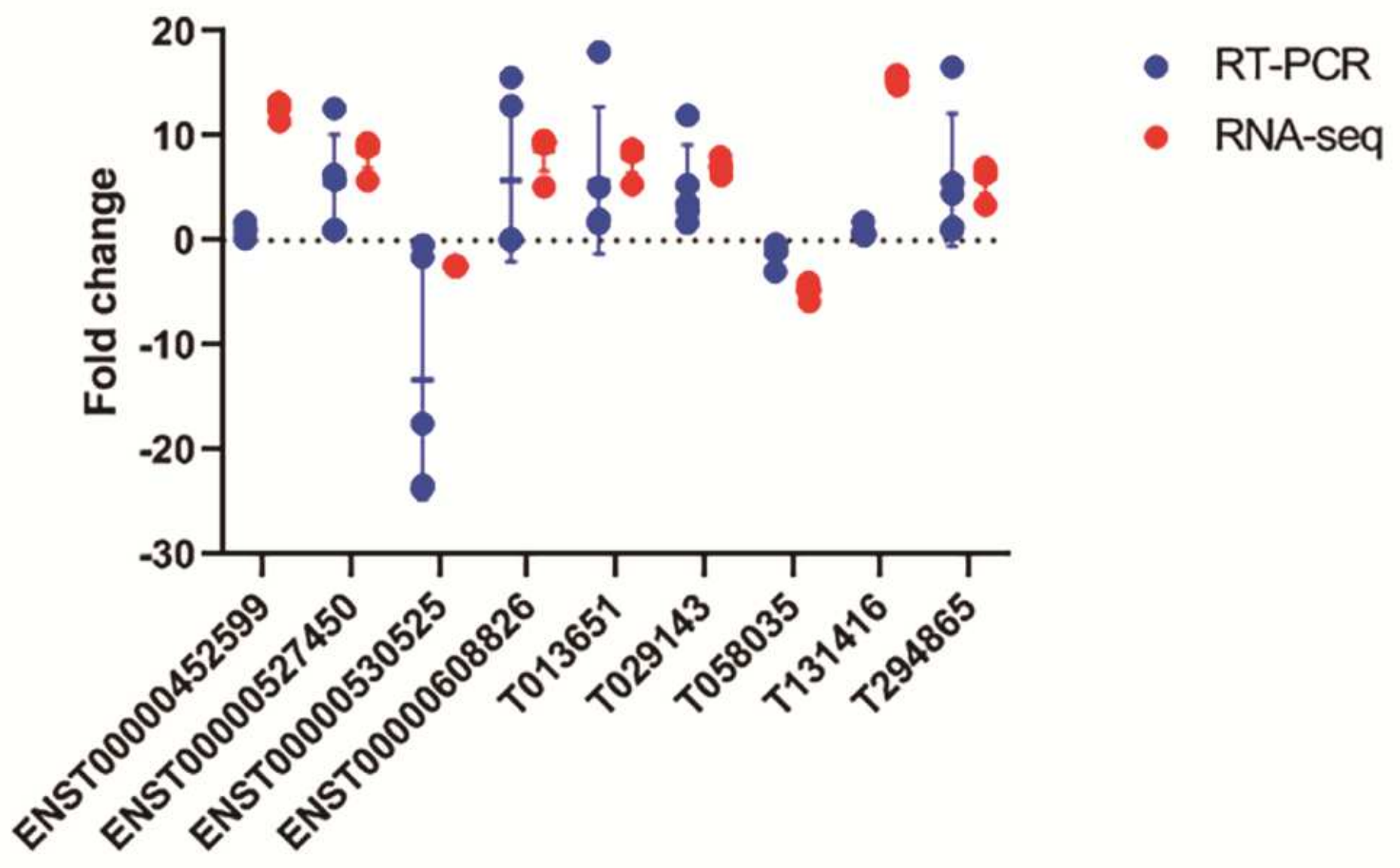

Figure 2

Validation of differential IncRNA expression. The expression level of the selected IncRNAs in the stroke group and non-stroke group. GPCR was used to verify differentially expressed IncRNAs $(p<0.05)$. The blue points represent qPCR and the red points represent the gene chip of RNA-seq. $n=6$. 


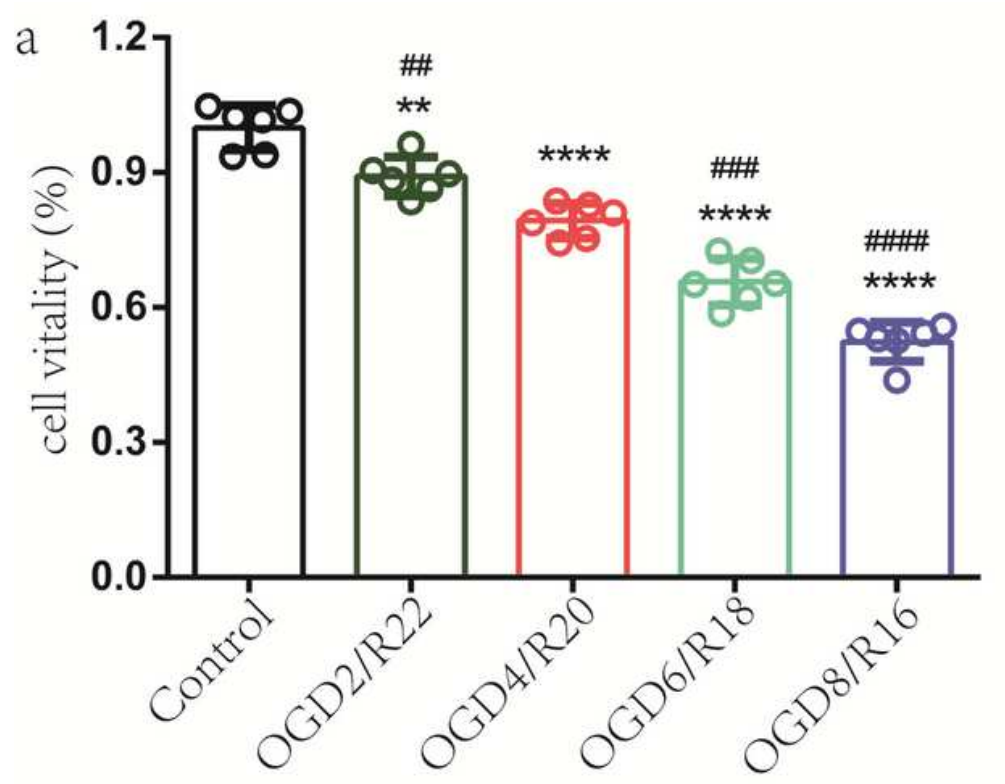

b

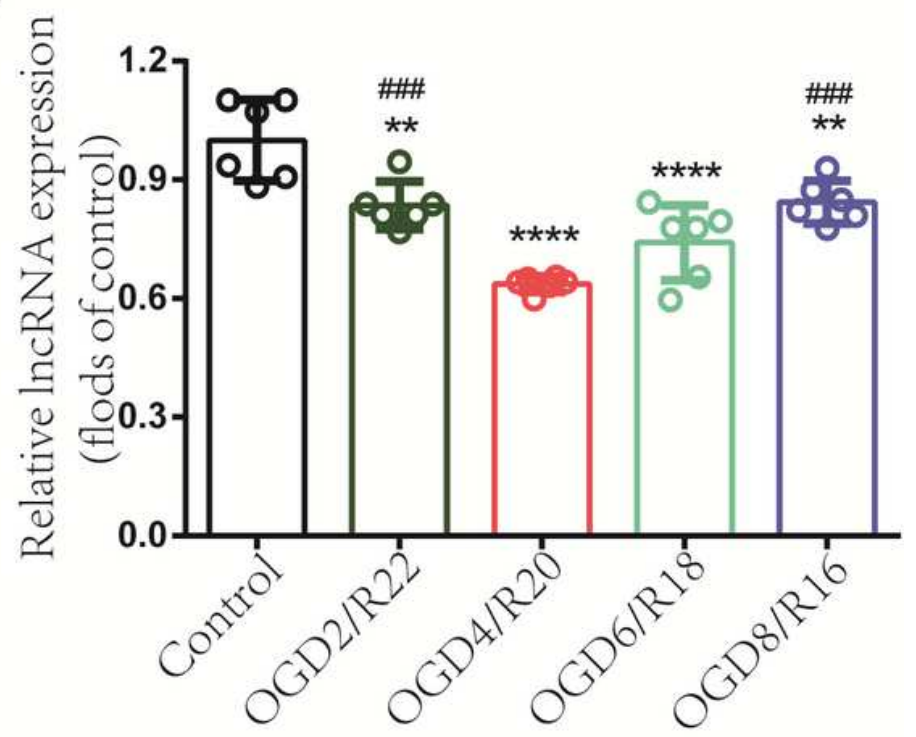

Figure 3

LncRNA ENST00000530525 is down-regulated in hCMEC/D3 after OGD/R. Cells were exposed to OGD for $2,4,6$ or $8 \mathrm{~h}$ and returned to room air (reoxygenation) for $22,20,18$ or $16 \mathrm{~h}$. Each bar is labeled with the duration of OGD followed by the period of reoxygenation (e.g.,OGD2/R22 refers to $2 \mathrm{~h}$ of OGD and 22h of reoxygenation).(a)The cell vitality was get more and more worse while the OGD time was rising.(b)The IncRNA highly down-regulated on the condition of OGD4/R20. For each treatment, the data are obtained

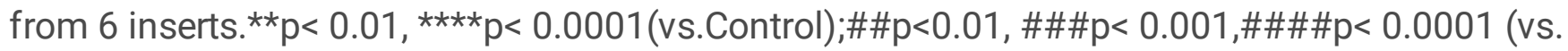
OGD4/R20),n=6,one-way ANOVA. 
a
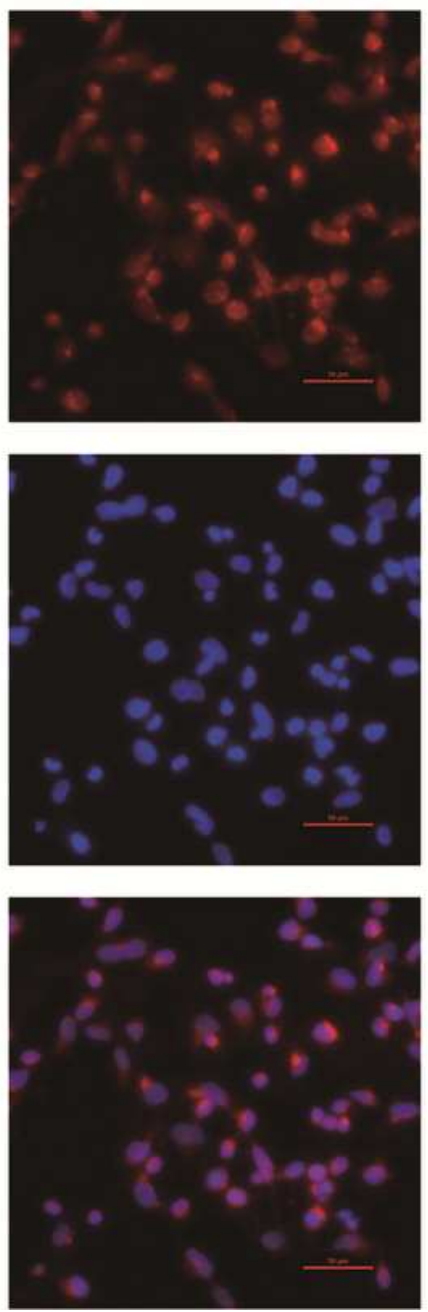

$18 \mathrm{~S}$
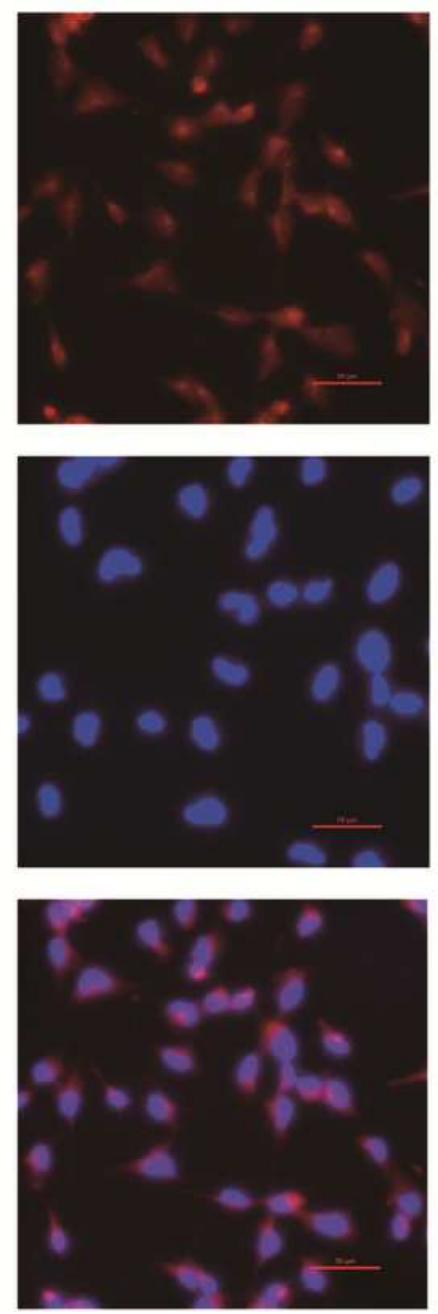

b

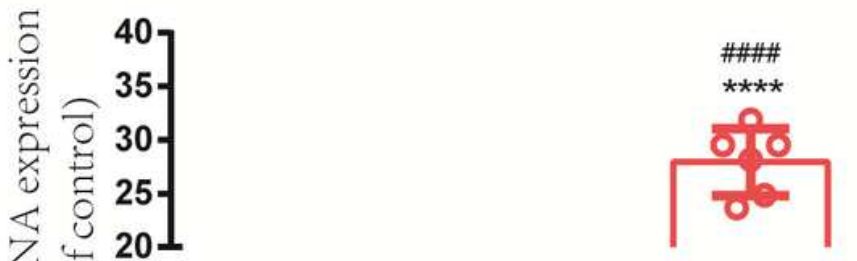

2.0

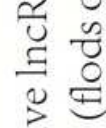

$\frac{3}{2}$

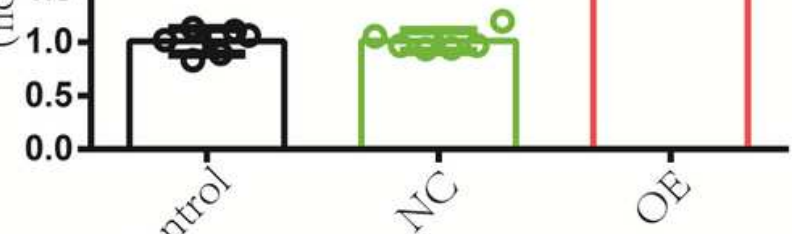

C

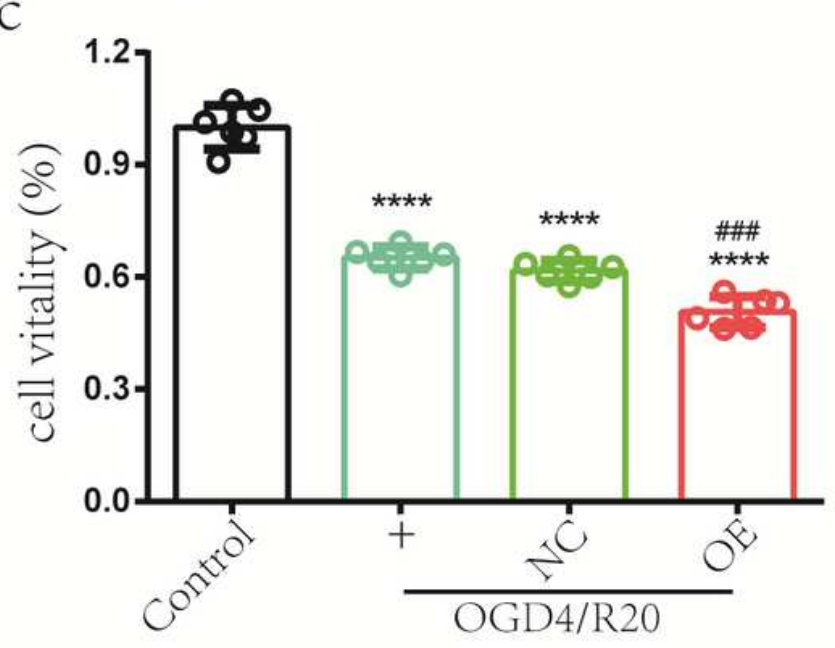

Figure 4

Up-regulating the Expression of IncRNA ENST00000530525 Affects the cell vitality of hCMEC/D3.(a)FISH showned that ENST00000530525 mainly distributed in the cytoplasm as the control group-18S.Scale bar:

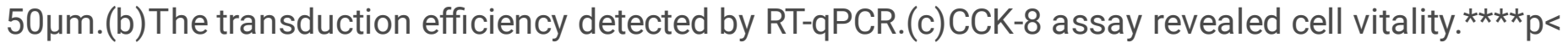
0.0001 (vs.Control);\#\#\#p<0.001,\#\#\#\#p 0.0001 (vs. NC), n=6,one-way ANOVA. 

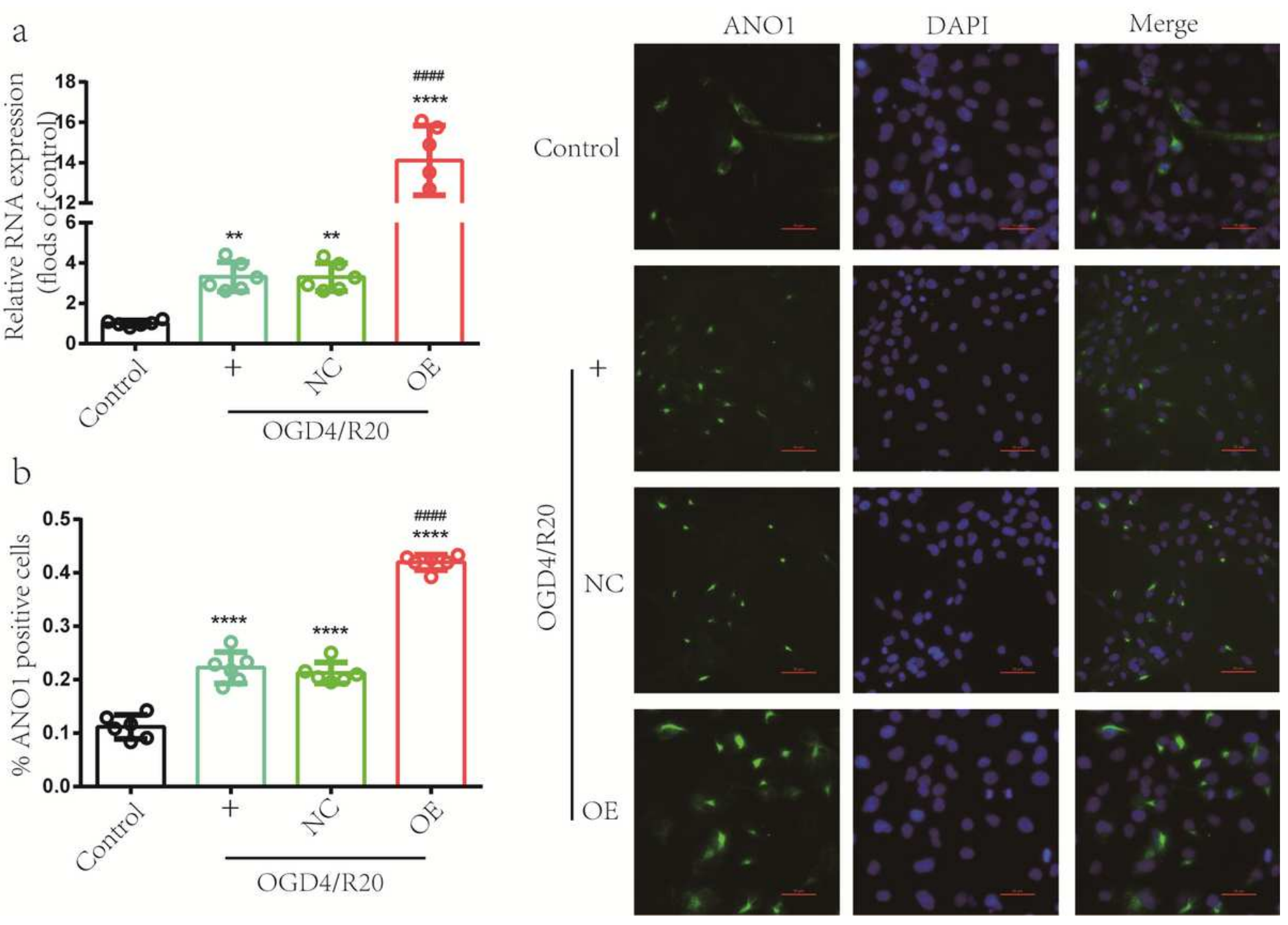

Figure 5

Up-regulating IncRNA ENST00000530525 increased the expression of AN01.The effects of IncRNA ENST00000530525 up-regulation by plasmid on gene and protein expressions of ANO1 in hCMEC/D3 after OGD4/R20 condition. (a)RT-qPCR results show that ANO1 increased after OGD4/R20, which increased more significantly when IncRNA ENST00000530525 was up-regulated by plasmid.(b)The immunofluorescence results show that transfecting a plasmid in hCMEC/D3 resulted in increasement of AN01 protein expressions. ${ }^{\star \star} p<0.01,{ }^{\star \star \star \star} p<0.0001$ (vs.Control);\#\#\#\#p<0.0001 (vs. NC), $n=6$,one-way ANOVA. 

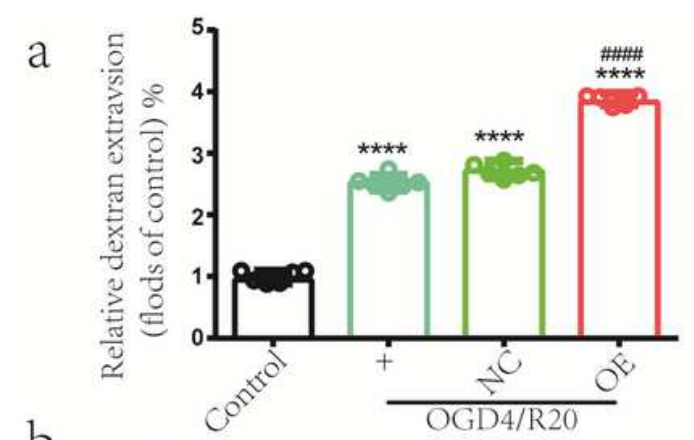

$\mathrm{b}$

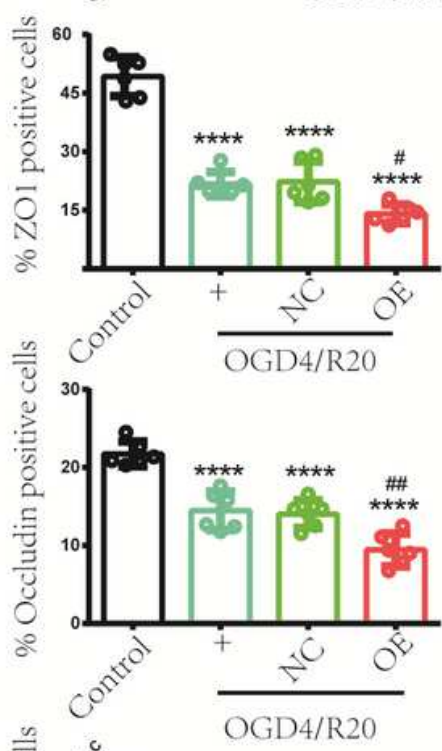

d

$\mathrm{C}$

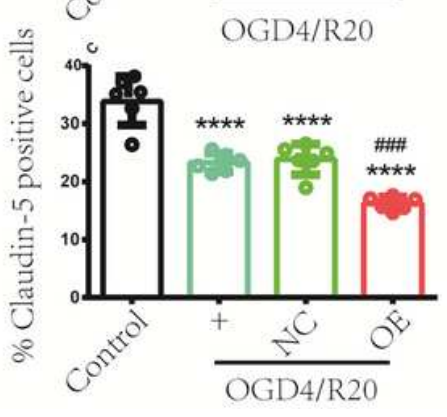

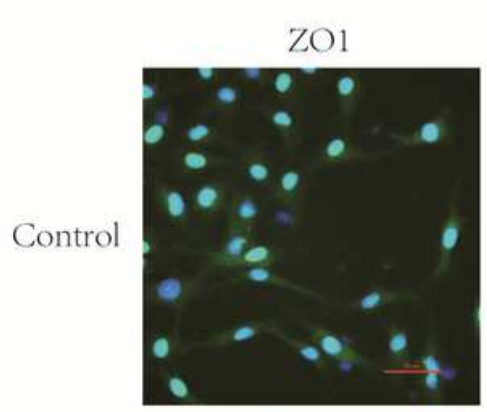
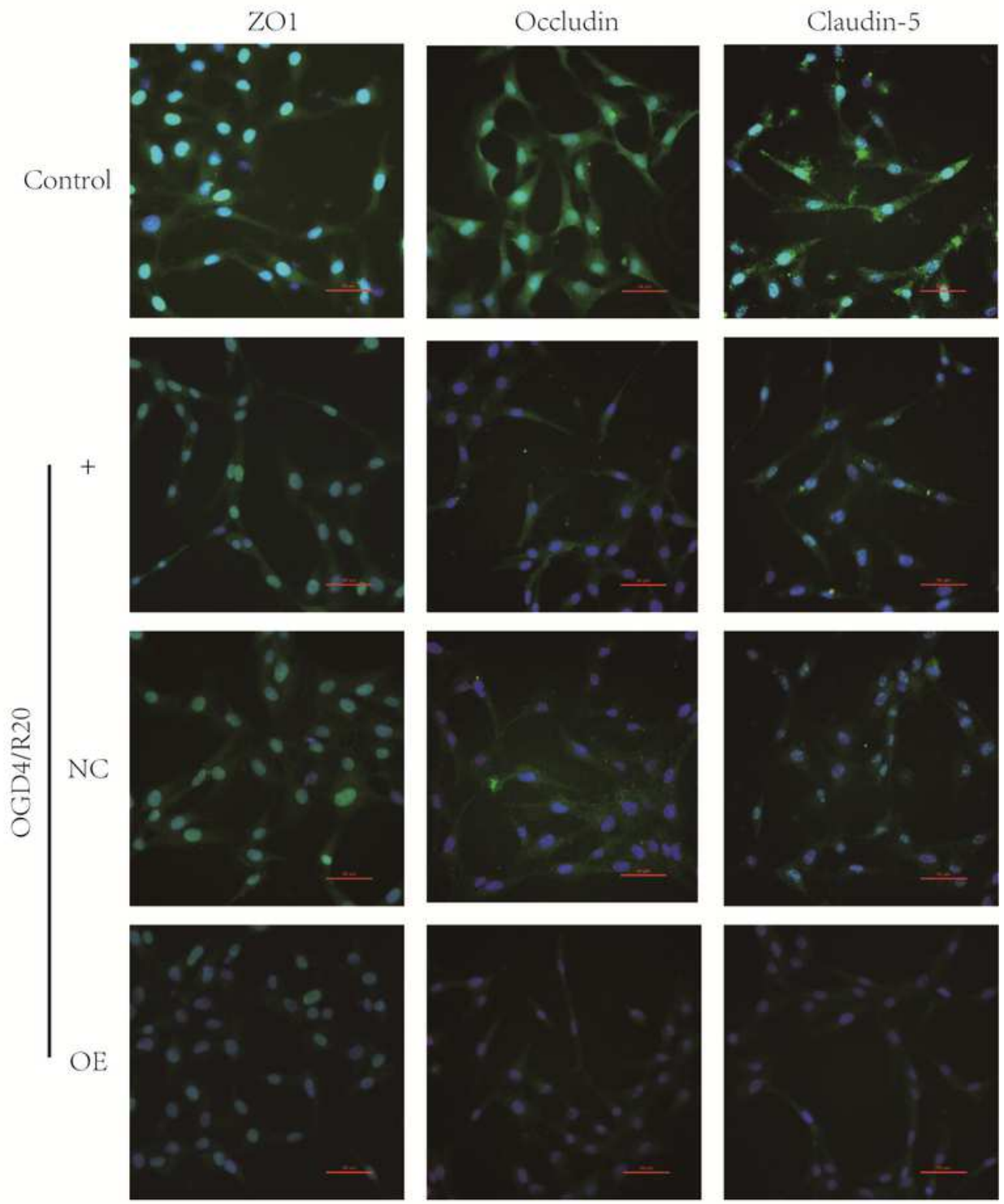

Figure 6

Increasing IncRNA ENST00000530525 deteriorated OGD/R-induced transendothelial permeability. (a)Quantification of transendothelial permeability detected by dextran leakage.(b-d)Representative immunofluorescence results of ZO-1, occludin and claudin-5 after OGD/R treatments. .*** $\mathrm{p}<$ 0.0001 (vs.Control); $\# p<0.001$, \#\#p< 0.0001;\#\#\#p<0.001,\#\#\#\#< 0.0001 (vs. NC), $n=6$,one-way ANOVA. 


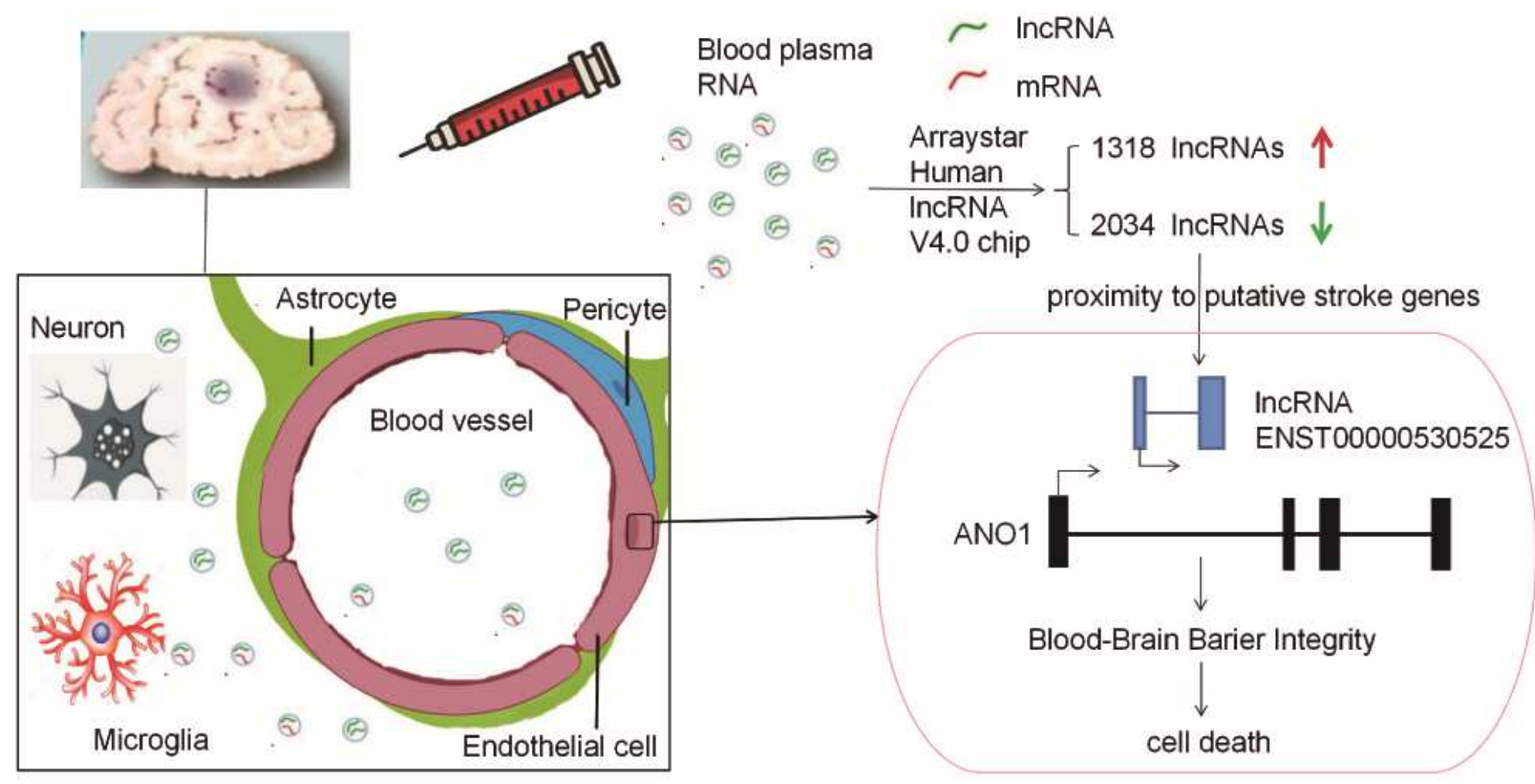

\section{Figure 7}

Schematic diagram of this paper.This study was performed to identify differentially expressed IncRNAs using a IncRNA microarray in whole blood amples of patients suffered from acute cerebral ischemia.There were 3352 IncRNA transcripts differently expressed between the blood plasma of stroke and non-stroke patients, with 1318 up-regulated and 2034 down-regulated IncRNAs using arraystar Human IncRNA V4.0 chip.Meanwhile the IncRNA ENST00000530525 was the highly down-regulated IncRNA and ANO1 was its proximity to putative stroke gene.Up-regulating IncRNA ENST00000530525 would improve the expression of ANO1, which would destroy BBB integrity further,in hCMEC/D3 after OGD4/R20.

\section{Supplementary Files}

This is a list of supplementary files associated with this preprint. Click to download.

- SupplementaryTableandFigure.pdf 